\title{
University student conceptual resources for understanding forces
}

\author{
Amy D. Robertson, ${ }^{1}$ Lisa M. Goodhew, ${ }^{2}$ Rachel E. Scherr, ${ }^{1}$ and Paula R. L. Heron ${ }^{2}$ \\ ${ }^{I}$ Physics Department, Seattle Pacific University, 3307 Third Ave W, Seattle, WA, 98199 \\ ${ }^{2}$ Physics Department, University of Washington, 3910 Fifteenth Ave NE, Seattle, WA, 98195
}

\begin{abstract}
We present preliminary results from our investigation of introductory physics students' conceptual resources for understanding forces. We analyzed a total of 1057 student responses to conceptual questions about forces and identified three common, prevalent resources that students used in justifying their answers, including the ideas that forces change the motion of objects, objects that have motion keep that motion, and motion is due to an imbalance of forces. We illustrate some of the ways in which these resources manifested in student responses and discuss how these ideas are continuous with physics understandings. We situate our work in the literature on student thinking about forces and instructor pedagogical content knowledge (PCK).
\end{abstract}

\section{INTRODUCTION}

Researchers' conceptualizations of PCK - i.e., what instructors need to know in order to teach - include both content knowledge and knowledge of students' ideas (KSI) in particular content areas [1,2]. Historically, KSI has been framed in terms of student misunderstandings [1,2], and research has contributed to instructor KSI by identifying common, incorrect patterns of student reasoning about particular content (e.g., [3]).

This is true of the extensive literature documenting student thinking about forces, within which the most commonly discussed idea is that of an impetus force [4-11]. Clement [8] defines this idea as the "belief that there is a force inside a moving object that keeps it going and causes it to have some speed" and that "fade[s] away as the object moves along." The literature depicts a number of different patterns in student responses that tie to the impetus idea:

- Students reason as though forces are necessary to sustain motion or that motion implies force [4,5].

- Students associate force with velocity, rather than acceleration [4-7]. This takes many forms, including: if there is no motion there is no force [4-6]; if there is no force there is no motion [7]; and the magnitude of the force is proportional to the speed $[6,7]$.

- Students treat forces as things that objects have and can transfer, rather than interactions between objects $[4,5]$.

The primary orientation toward students' ideas embodied by this literature is misconceptions-, difficulties-, or misunderstandings- oriented (e.g., [7,9-11]): these papers focus on students' incorrect ideas, position students' ideas against the canon, and/or suggest that students' ideas should be corrected during formal instruction.

Our research instead centers on identifying some of the common, prevalent resources that students use to understand forces. Rather than framing student thinking as discontinuous with formal physics, or as in need of correction, we frame student thinking as continuous with formal physics, as something to be built on. Our aim is to broaden instructors' KSI - to supplement the existing literature focusing on student misunderstandings about forces with patterns in student thinking that represent resources for understanding forces. In this paper, we report three common, prevalent resources that students used to justify their answers to questions about forces.

One may argue that this kind of KSI is already available to instructors; they could simply re-interpret the existing literature in terms of productive possibilities. However, this literature represents researchers' interpretations of student responses to conceptual questions about forces, and these interpretations were primarily informed by the lens of what students were struggling with, and/or in what ways student ideas were inconsistent with the canon. As we will elaborate in our Discussion, taking a resources orientation toward the data itself affects what we see, including how we interpret what students mean by what they say. The extent to which using a different lens reveals entirely new categories of student thinking is an empirical question that, to our knowledge, has not yet been answered at scale.

Further, our aim is to influence the ideas that instructors see [12] and the ways in which instructors frame student ideas, by identifying some of the common, prevalent resources that students use in the context of forces. When instructors elicit ideas from their students, what they will receive is closer to the raw data that we are using to inform our work than to the interpretations of student thinking offered by the existing literature. Our aim is to support instructors in organizing their "data" into resources; thus, it bears on us to both (1) identify what they might expect to see, resources-wise (i.e., identify common, prevalent resources for understanding forces within introductory physics student responses) and (2) illustrate what these resources "look like" in student responses to conceptual questions. Again, here, the extent to which these resources - and what they look like, in practice - maps directly onto re-framings of existing interpretations of student thinking is an empirical question, one that we will return to in the Discussion.

\section{THEORETICAL FRAMEWORK: RESOURCES}

Here, we define a resource as an idea that could represent a beginning of more sophisticated scientific understandings, consistent with [13-15]. Our orientation toward student thinking is consistent with that embodied in [16]: we assume that students think in ways that are sensible to them; that 
student thinking is often continuous with expert thinking in physics; and that quality learning builds on students' ideas.

Theoretical accounts of resources depict knowledge as dynamic and context-dependent [17], such that resources that are activated in one context are not necessarily expected to be activated in another. In this project, we report common, prevalent resources - those that come up in multiple questions, for many students. This choice is informed by our aim to provide instructors with a sense of what resources they might expect their students to use, in a typical introductory physics course. In the absence of a representative sample of introductory physics students, we draw on a model of generalizability that emphasizes recurrence: "how consistently a particular [pattern or relationship] reproduces across multiple sources of heterogeneity" [18]. Recurrenceoriented research is not typical of a resources theoretical framework, but the theory allows for resources or networks of resources that are activated in multiple contexts [17], so long as they are understood to manifest differently in different contexts. Drawing on this theoretical framework and considering our aims, we both (a) frame the resources we report as those that may be likely to be activated in forces contexts and (b) illustrate the variety of student responses that instantiate these resources in different contexts.

\section{RESEARCH METHODS}

\section{A. Question development}

The resources we report in this paper were identified in the context of student responses to five conceptual questions about forces, all depicted in Fig. 1. Many of these are adapted from existing questions that have guided the misunderstandings-oriented literature described in the Introduction. For example the pendulum, modified coin toss, and airplane questions are modified or reproduced from [911], respectively. The ontologies question is original but is based on ideas reported in the literature. We modified or re- used existing questions in part to illustrate how the resources framework can support instructors in reframing student thinking, even in contexts in which misunderstandings are clearly visible, and in part because we suspected that understanding the sense-making behind students' incorrect answers would point us toward resources. The furniture question is original, and is meant to invite students to sensemake about forces in a familiar context. Five validation interviews were conducted for each question.

\section{B. Sample}

A total of 1057 students in calculus-based introductory physics courses from 4 universities across the US answered our questions. To be included in this paper, at least $70 \%$ of the students in a given sample had to submit their answers, with written reasoning, to the relevant question. Students received homework or pre-lab points for their responses.

\section{Identification of resources}

Robertson and Goodhew used student responses to the five questions to create an emergent coding scheme [19] that described the resources students were using to reason about forces. We conducted preliminary analyses of student responses to each question, articulating ways in which students were reasoning that we could frame as continuous with scientific understandings. We used our preliminary analyses to identify patterns in student reasoning across contexts (and for many students), and these ultimately became the codes in our scheme. Examples of these codes and illustrative student responses are given in Section IV.

\section{Coding}

Robertson and Goodhew then independently coded each student response. A single student response was often assigned more than one code to capture the full range of resources represented therein. For each student response, we

\begin{tabular}{|c|c|c|}
\hline \multicolumn{2}{|c|}{$\begin{array}{l}\text { Pendulum question: } \\
\text { A pendulum bob attached to a string moves back and forth in the } \\
\text { trajectory outlined by the dotted lines in the pictures below. The } \\
\text { pendulum is moving to the right at instants } A \text { and } B \text { and is at rest at } \\
\text { instant } C \text {; the arrows indicate the velocity of the pendulum in each }\end{array}$} & $\begin{array}{l}\text { Airplane question: } \\
\text { A bowling ball accidentally falls out of the cargo bay of } \\
\text { an airliner as it flies along in a horizontal direction. As } \\
\text { seen from the ground, which path would the bowling } \\
\text { ball most closely follow after leaving the airplane? } \\
\text { Explain why your answer makes sense to you. }\end{array}$ \\
\hline $\begin{array}{l}\text { instant. Draw the trajectory } \\
\text { of the pendulum bob after } \\
\text { the string is cut for each } \\
\text { case }(A \text { through } C) \text {, and } \\
\text { explain your reasoning. }\end{array}$ & & $\begin{array}{l}\text { Furniture question: } \\
\text { You may have experienced that when you're trying to push a heavy box or piece of } \\
\text { furniture across the floor, it's harder to get it moving than to keep it going once it's } \\
\text { already moving. How do you make sense of this, given what you know about forces? } \\
\text { (There's no right or wrong answer here; this question is about writing down what } \\
\text { makes sense to you.) }\end{array}$ \\
\hline $\begin{array}{l}\text { Modified coin toss question: } \\
\text { A stecl ball is tossed from point } A \text { straight } \\
\text { up into the air and caught at point } E \text {, as in } \\
\text { the figure at right. In the boxes below, draw } \\
\text { one or more arrows showing the direction of } \\
\text { each force acting on the coin when it is at } \\
\text { points } B \text { and } D \text {. Explain your reasoning. }\end{array}$ & $\frac{\mathrm{A}}{\text { ball moving up }} \frac{\downarrow E}{\frac{\downarrow E}{\text { tall moving down }}}$ & $\begin{array}{l}\text { Ontologies question: } \\
\text { Which of the following statements is true in at least some situations? Mark all that } \\
\text { apply. For each option that you mark as true in at least some situations, give an } \\
\text { example of a situation in which that statement applies. } \\
\text { a. A force is used up. } \\
\text { b. One object has more force than another. } \\
\text { c. A force is transferred from one object to another. } \\
\text { d. A force is an interaction between objects. }\end{array}$ \\
\hline
\end{tabular}

FIG 1. Conceptual questions. The pendulum question was adapted from Cognition, Vol. 9, A. Caramazza, M. McCloskey, and B. Green, "Naïve beliefs in 'sophisticated' subjects: misconceptions about trajectories of objects," pg. 7, 1981, with permission from Elsevier. The modified coin toss question was adapted from J. Clement, "Students' preconceptions in introductory physics," American Journal of Physics 50(1), pgs. 66-71 (1982). The airplane question was reproduced from D. Hestenes, M. Wells, and G. Swackhamer, "Force concept inventory," The Physics Teacher 30, pgs. 141-158 (1992). 
compared our individually assigned codes and kept only those that were assigned by both coders, such that the final codes reflected $100 \%$ agreement between coders. We used these final codes to calculate the percentages of student responses that reflected each resource.

As a measure of inter-rater agreement [20], we took the normalized difference between the total number of possible codes and the total number of (original) disagreements between the two coders. For example, given our three codes, if Robertson assigned student $x$ 's response codes $\mathrm{A}$ and $\mathrm{B}$ and Goodhew assigned it codes $\mathrm{A}$ and $\mathrm{C}$, there would be two disagreements and three possible codes for this data set of one response, giving a percentage agreement of 33\%. Our percentage agreement for the full data set was $92 \%$.

\section{UNIVERSITY STUDENT CONCEPTUAL RESOURCES FOR UNDERSTANDING FORCES}

In this section, we describe and illustrate three resources for understanding forces that we identified in student responses to the five questions introduced in Section III. Table I documents the percentages of student responses reflecting each resource for each question and sample.

TABLE I. Percentages of student responses reflecting each resource

\begin{tabular}{|c|c|c|c|}
\hline \multirow[t]{2}{*}{ Question/sample } & \multicolumn{3}{|c|}{$\begin{array}{l}\text { Percentage of responses } \\
\text { reflecting resource }\end{array}$} \\
\hline & $\mathrm{A}$ & B & $\mathrm{C}$ \\
\hline \multicolumn{4}{|l|}{ Airplane question } \\
\hline WWU $(N=230)$ & $40 \%$ & $18 \%$ & $0 \%$ \\
\hline $\operatorname{MSU}(N=84)$ & $38 \%$ & $50 \%$ & $0 \%$ \\
\hline \multicolumn{4}{|l|}{ Ontologies question } \\
\hline WWU $(N=263)$ & $26 \%$ & $0 \%$ & $3 \%$ \\
\hline $\operatorname{MSU}(N=81)$ & $14 \%$ & $0 \%$ & $1 \%$ \\
\hline \multicolumn{4}{|l|}{ Furniture question } \\
\hline WWU $(N=251)$ & $0 \%$ & $11 \%$ & $29 \%$ \\
\hline $\operatorname{MSU}(N=84)$ & $0 \%$ & $12 \%$ & $23 \%$ \\
\hline \multicolumn{4}{|l|}{ Coin toss question } \\
\hline $\mathrm{UW}(N=355)$ & $26 \%$ & $0 \%$ & $5 \%$ \\
\hline \multicolumn{4}{|l|}{ Pendulum question } \\
\hline $\operatorname{SPU}(N=63)$ & $43 \%$ & $32 \%$ & $0 \%$ \\
\hline $\mathrm{UW}(N=292)$ & $68 \%$ & $50 \%$ & $0 \%$ \\
\hline
\end{tabular}

\section{A. Forces change the motion of objects.}

Table I indicates the percentages of student responses that can be interpreted as drawing on the resource "forces change the motion of objects." These responses often identified a force as the reason for a change in an object's speed and/or direction of motion, or identified a change in motion and inferred the presence of a force. For example, in the airplane question, a student chose answer (a), writing, "The bowling ball would most likely follow the path A because the ball will experience air resistance as it is dropped. The air resistance will pull the ball back making it follow this trajectory." Here, the student identifies a change in the bowling ball's motion (it moves backward) and attributes this change to a force (air resistance). In the ontologies question, one student chose (c) and wrote, "[A] pool cue transfers its force to the cue ball which moves in a direction." This student also attributes a change in the cue ball's motion (rest to moving) to a force from the pool cue.

Though both responses are canonically incorrect, we see them as continuous with formal physics, in drawing on the resource that forces change the motion of objects. We see this resource as continuous with both Newton's first and second laws - i.e., as consistent with the idea that net force is related to acceleration and that objects continue in their state of motion unless acted on by a net force.

\section{B. Objects that have motion keep that motion.}

When students were asked to draw or identify the trajectory of a moving object after it has been released, many justified choices that maintain a component of the object's original motion in terms of this resource - that objects that have motion keep that motion. For example, one student chose trajectory (c) in the airplane question and wrote, "The path would follow the line of $\mathrm{C}$. This is because the ball still has positive momentum and will continue to move forward after the ball has left the plane." This student identifies a trajectory that maintains the "forward" motion of the bowling ball, arguing that the ball "still has" - some of its original motion. Another student drew a parabolic trajectory for the pendulum bob in the pendulum question, saying, "the ball would move in the direction of the velocity vector and move downward since the ball's initial direction of movement when it was cut is the velocity shown in case A. It would also move downward due to acceleration from gravity." This student justifies their drawing of a trajectory that blends horizontal and vertical motion in terms of the continued motion along "the ball's initial direction of movement" and its vertical acceleration due to gravity.

In both of these examples - again, the first of which is canonically incorrect - students are drawing on the resource that an object in motion will maintain its motion, an idea that is continuous with Newton's first law.

\section{Motion is due to an imbalance of forces.}

Table I reflects the percentages of student responses that attributed movement to an imbalance of forces and/or argued that such an imbalance is necessary to make an object move or accelerate. For example, in the furniture question, one student wrote, "You know that the forces acting on the heavy box would be the gravitational force pushing downwards, the normal force of the floor pushing up, and the friction would be pushing against the box. When you begin to push the box you will cause an unbalance in those forces. At rest though, the forces acting on the box are at equilibrium and it is harder to get the box moving because you need to break the 
equilibrium of the forces." This student argues that it is harder to get the box moving because you have to "break the equilibrium of the forces" and that the movement of the box is due to an "unbalance in those forces." In the modified coin toss question, one student drew a normal force upward and a weight force downward at point B and a single weight force downward at point D, writing, "The ball being thrown in the air is moving against gravity at point $\mathrm{B}$. At point $\mathrm{D}$ the ball is falling so [the] weight force is overcoming the normal force so the ball falls." This student justifies the downward motion of the ball in terms of an imbalance of forces: the weight force "overcame" the normal force so the ball falls.

In each of these examples, we see students attributing motion - starting, changing, and in some cases maintaining motion - to an imbalance of forces, often reflected in terms of "unbalanced" or "overcoming." We see these ideas as continuous with Newton's second law - that forces "give rise" to acceleration [21].

\section{DISCUSSION}

In this paper, we describe three conceptual resources for understanding forces, identified in introductory physics students' responses to five questions. The primary aim of this research is to (a) support instructors in framing student thinking as continuous with scientific understanding, offering examples of what this can look like, and, in doing so, to (b) broaden instructors' KSI in the context of forces.

In the Introduction, we framed the novelty of this research, as compared to existing (misunderstandingsoriented) literature on student ideas about forces, as an empirical question: To what extent to will using the lens of resources reveal new categories of student thinking than those articulated in the existing literature? Though we cannot answer this question at this exploratory phase of our work, we do think that the three resources we articulate in Section IV constitute more than re-interpretations of the impetus-like ideas reported in the literature. In particular, the impetus force idea, according to Clement [8], is the "belief that there is a force inside a moving object that keeps it going and causes it to have some speed." According to the literature, students instantiate this idea, e.g., when they reason that forces are necessary to sustain motion or that motion implies force $[4,5]$. In analyzing student responses in our sample with a resources lens, however, we see a more nuanced and/or disciplinarily-appropriate meaning that students associate with the idea of forces. For example, rather than treating force as an impetus that resides in an object and fades away as it moves, students treat forces as agents that affect motion; they acknowledge the persistence of motion in situations in which a force ceases to act on an object; and they articulate the relationship between imbalance or disequilibrium and changes in motion. These interpretations of student responses not only re-frame student thinking as continuous with formal physics; they help us to see the disciplinarily-sophisticated meanings that students do associate with forces. These meanings are what we wish to make visible to physics instructors; we want them to see what students are doing, not only to re-interpret what students are misunderstanding.

\section{ACKNOWLEDGEMENTS}

This material is based upon work supported by National Science Foundation Grant No. 1608510. We are grateful for colleagues who collected data for us, including D. Bolton, A. Boudreaux, M. Caballero, K. Gray, E. Sayre, and L. Seeley.
[1] L.S. Shulman, Harvard Educ Rev 57, 1 (1987).

[2] D.L. Ball, M.H. Thames, and G. Phelps, J Teach Educ 59, 389 (2008).

[3] http://www.ipn.uni-kiel.de/aktuell/stcse/. Retrieved $6 / 21 / 2017$.

[4] http://www.diagnoser.com/teacherapp/home\# resources/subject/SU-0. Retrieved 6/21/2017.

[5] A.C. Alonzo and J.T.Steedle, Sci Educ 93, 389 (2009).

[6] R. Gunstone and M. Watts, in Children's Ideas in Science, edited by R. Driver (Open University Press, Philadelphia, 1985), p. 85.

[7] A.B. Champagne, L.E. Klopfer, and J. H. Anderson, Am J Phys 48, 1074 (1980).

[8] J. Clement, in Proceedings of the International Seminar: Misconceptions in Science and Mathematics, edited by H. Helm and J.D. Novak (Cornell University Press, New York, 1983), p. 310.

[9] A. Caramazza, M. McCloskey, and B. Green, Cognition 9, 117 (1981).

[10] J. Clement, Am J Phys 50, 66 (1982).
[11]D. Hestenes, M. Wells, and G. Swackhamer, Phys Teach 30, 141 (1992).

[12] C. Goodwin, Am Anthropol 96, 606 (1994).

[13] D. Hammer, F. Goldberg, and S. Fargason, Rev Sci, Math, ICT Educ 6, 51 (2012).

[14] D. Hammer, Cognition Instruct 15, 485 (1997).

[15] B.W. Harrer, V.J. Flood, and M.C. Wittmann, Phys Rev PER 9, 023101-1 (2013).

[16] A.A. diSessa, Cognition Instruct 10, 105 (1993).

[17] D. Hammer, et al., in Transfer of Learning from a Modern Multidisciplinary Perspective, edited by J.P. Mestre (Information Age Publishing, Greenwich, 2005), p. 89.

[18] T.D. Cook, Educ Eval Policy An 24, 175 (2009).

[19] K. Krippendorff, Content Analysis: An Introduction to Its Methodology (Sage, Thousand Oaks, 2013).

[20] R.E. Scherr, K.P. Gray, M.C. Wittmann, and S. Vokos, Phys Rev PER (in preparation).

[21] D.C. Giancoli, Physics for Scientists and Engineers, (Prentice Hall, Upper Saddle River, 2000). 